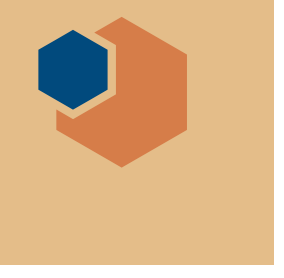

\title{
Innovate UK designed to better attract businesses
}

\author{
www.innovateuk.gov.uk
}

$T^{3}$ he United Kingdom's Innovate UK, the country's biggest source of government backing for research and development (R\&D), mixes materialsspecific programs with general support for industry sectors.

With a budget of more than $£ 560$ million (approx. USD $\$ 800$ million) a year, Innovate UK has grown to become the main source of public support for R\&D in the United Kingdom. When the UK's Technology Strategy Board (TSB) rebranded itself as Innovate UK two years ago, the organization was acknowledging changes in its role since it started as a small but influential committee with a small budget.

Drawn mostly from industry, TSB advised the UK government on its support for technology and innovation. Set up in 2004 as an internal body within the government department responsible for innovation, the TSB was a small player on the R\&D scene. Its initial budget was just $£ 150$ million (USD $\$ 210$ million) over three years.

The organization, which became an independent body in 2007, has invested around $£ 1.8$ billion (USD $\$ 2.5$ billion) in innovation over the past decade. It claims that this spending was "more than matched by the private sector." Along with much of the UK government's support for research, Innovate UK has maintained its budgets at a time of large cuts in public spending.

It is not just the level of spending and the name of the organization that have changed over the years; more recently the focus has shifted from technologies to "sector groups." These currently include emerging and enabling technologies, manufacturing and materials, and health and life sciences. This new emphasis contrasts with the more generic areas that TSB had backed, such as advanced materials, electronics and photonics, and information communication and technology.

The change in emphasis is designed in part to make it easier for companies, especially small- and medium-size enterprises (SMEs), to understand, with the hope that more companies will bid for backing. "We are trying to make our programs a lot simpler," says Zoe Webster, Head of High Value Manufacturing at Innovate UK. Webster explains that the organization sees the sector group for manufacturing and materials as "a main home for materials innovation" under the new structure.

The simplification has also led to changes in how Innovate UK runs the competitions that are at the heart of its process for selecting projects to back. Companies can submit proposals for grants for research projects that last between six months and three years and cost from $£ 50,000$ to $£ 2$ million. Each project must have at least two partners, including a SME, and be led by a business. It can, though, include research organizations such as universities.

Innovate UK's manufacturing and materials sector group plans to invest up to $£ 15$ million in its current competition, with a sec ond competition due later this year. The organization hopes that the new simpler system, with more general calls for entries rather than defined calls for particular technologies, will also make it easier for businesses to have their voices heard. "We have got a great opportunity to have businesses tell us what they need to do to innovate going forward in manufacturing and materials," says Webster.

The current competition is open to bids from all areas of 
manufacturing and materials. However, Innovate UK is nudging applicants in a couple of directions. For example, Webster hopes to encourage submissions in "design for manufacture, use and end of life."

Innovate UK is also keen to encourage companies to think about what has become known as Industry 4.0. "We are particularly interested in projects with strong elements of digital innovation," says Webster. As the competition brief describes it, in this round they are looking for "the novel application of a digital technology or approach to manufacturing or materials development (such as automation, modeling and simulation, data analysis)."

"We see a number of manufacturers talking about Industry 4 but not really knowing what it means for them," says Webster. "So this is really to give SMEs the space to start exploring those issues and to really highlight the importance of it for them."

Innovate UK does not rule out funding technologies that have been a part of earlier competitions. "We are obviously still interested in 'lightweighting,' materials for energy, energy generation and storage," she adds.

Innovate UK sees its gentle nudging toward particular topics as part of its remit to encourage developments in areas that it sees as of growing importance to the UK's economy. "There is still huge value for us as thought leaders to recognize key strategic areas for the UK and to move forward," says Webster. "That is something we will still be doing," she adds. "However, we recognize that for businesses if they have got a new idea, if they have to wait for the right competition to come along, either in the right technical area or in the right size for what they wanted to do, that is not particularly helpful for them."

While the emphasis of Innovate UK is funding projects through competitions, it also works alongside and funds Knowledge Transfer Networks (KTNs). Among other things, the Materials KTN helps to organize briefing events for competitions. Through its groups, it also acts as a broker, bringing companies and research organizations together to form project consortia. "For people who want to find project partners," says Webster, "they can contact the Knowledge Transfer Network, which has thousands of contacts and is very good at doing that kind of matchmaking."

Innovate UK also provides core funding for a series of Catapults. These describe themselves as "a network of world-leading centers designed to transform the UK's capability for innovation in specific areas and help drive future economic growth." The high-value manufacturing catapult is the natural home for materials expertise, says Webster. "If a SME contacted them they might also have very good ideas for people they could talk to."

It is too soon to know if Innovate UK's reorganization will attract more SMEs to enter competitions. "We just don't know because it is a new way of doing it," says Webster. Previous competitions could bring in 500 or 600 entries, she explains. "I think it is reasonable to expect a few hundred," she adds. "But beyond that we just don't know."

Michael Kenward
India and UK to work as R\&D partners in solar alliance and nanomaterials research

$\mathrm{A}^{\mathrm{t}}$ $\mathrm{t}$ the 5 th Indo-UK Science and Innovation Council Meeting (SIC) held in London in June, the two countries agreed on collaboration in a number of projects, including areas in solar energy and nanomaterials. The meeting was cochaired by India's Minister for Science \& Technology and Earth Sciences (DST), Harsh Vardhan, and the UK Minister for Universities \& Science, Jo Johnson.

As part of their commitment to the solar alliance, the research projects will focus on systems-level design and development covering solar energy generation, storage systems, and grid integration, particularly for microgrid systems.

Furthermore, researchers from India will have access to the neutron scattering facility of the UK's Science and Technology Facilities Council at Rutherford Appleton Laboratory, Oxford. This will help to enhance Indian capability to fabricate structures at the nanoscale level for creating interesting and technologically important materials through research in fundamental science.

The SIC oversees the entire gamut of the India-UK science, technology, and innovation cooperation and meets once every two years. The last meeting of the SIC was held in New Delhi in November 2014, at which time the two countries launched the Newton-Bhabha Program to support bilateral science and technology (S\&T) cooperation. Both nations have now agreed to address grand societal challenges on food, energy, and water security; health and well-being; and smart cities and rapid urbanization with increasing access and sharing of expertise, resources, and facilities.

Under the Newton-Bhabha Program, the two sides also agreed on a collaborative program on energy efficiency in the built environment as a part of the Smart Cities mission. They agreed on augmenting the India-UK Centre of Advanced Manufacturing cooperation linked to the Make in India Program and UK's Catapult Centre Program. The projects will be designed to deliver industry-relevant results that can be readily applied to current challenges in the manufacturing supply chain.

A program on water quality research will address natural contaminants such as arsenic and fluoride in groundwater as well as other pollutants (e.g., pharmaceutical and personal care products). To further support industrial R\&D, the two countries added projects in the areas of clean technology, electronic design, and the Internet of things. 